\title{
Prenatal Exposure to Lamotrigine: Effects on Postnatal Development and Behaviour in Rat Offspring
}

\author{
Sekar Sathiya, ${ }^{1}$ Murugan Ganesh, ${ }^{2}$ Periyathambi Kalaivani, ${ }^{1}$ Vijayan Ranju, ${ }^{1}$ \\ Srinivasan Janani, ${ }^{3}$ Bakthavachalam Pramila, ${ }^{1}$ and Chidambaram Saravana Babu ${ }^{1}$ \\ ${ }^{1}$ Centre for Toxicology and Developmental Research (CEFT), Sri Ramachandra University, Chennai, Tamil Nadu 600116, India \\ ${ }^{2}$ Department of Biochemistry, Sri Ramachandra University, Chennai, Tamil Nadu 600116, India \\ ${ }^{3}$ School of Chemical and Biotechnology, Shanmugha Arts, Science, Technology and Research Academy (SASTRA University), \\ Thanjavur, Tamil Nadu 613402, India
}

Correspondence should be addressed to Chidambaram Saravana Babu; ceftpublications@gmail.com

Received 11 January 2014; Accepted 19 February 2014; Published 14 April 2014

Academic Editors: I. Berger, A. M. Depino, and R. Kulesza

Copyright $\odot 2014$ Sekar Sathiya et al. This is an open access article distributed under the Creative Commons Attribution License, which permits unrestricted use, distribution, and reproduction in any medium, provided the original work is properly cited.

\begin{abstract}
Use of antiepileptic drugs (AEDs) in pregnancy warrants various side effects and also deleterious effects on fetal development. The present study was carried out to assess the effects of prenatal exposure to lamotrigine (LTG) on postnatal development and behavioural alterations of offspring. Adult male and female Sprague Dawley rats weighing 150-180 g b. wt. were allowed to copulate and pregnancy was confirmed by vaginal cytology. Pregnant rats were treated with LTG (11.5, 23, and $46 \mathrm{mg} / \mathrm{kg}$, p.o) from gestational day 3 (GND 3) and this treatment continued till postnatal day 11 (PND 11). Offspring were separated from their dam on day 21 following parturition. LTG, at $46 \mathrm{mg} / \mathrm{kg}$, p.o, produced severe clinical signs of toxicity leading to death of dam between GND 15 and 17. LTG, at 11.5 and $23 \mathrm{mg} / \mathrm{kg}$, p.o, showed significant alterations in offspring's incisors eruption and vaginal opening when compared to age matched controls. LTG (23 mg/kg, p.o) exposed female offspring expressed hyperactive behaviour and decreased GABA-A receptor expression when compared to control rats. These results reveal that prenatal exposure to LTG may impart differential postnatal behavioural alterations between male and female rats which paves way for further investigations.
\end{abstract}

\section{Introduction}

Use of antiepileptic drugs (AEDs) during pregnancy presents the dilemma of minimising the risk of seizure and avoiding adverse effects in the unborn child. Various studies revealed that prenatal exposure of AEDs imparts serious effects on infants [1-3]. Further, prenatal exposures to AEDs were also reported to produce cognitive impairment $[4,5]$. Vigabatrin and valproate were reported to produce neuronal migration defect [6] upon prenatal exposure. Janz and Fuchs [7] reviewed that exposure to AED during pregnancy increases the risk of miscarriage and stillbirth rate. However, little attention was paid to the cellular effects of AEDs during postnatal development and in adulthood.

Lamotrigine (LTG), a phenyltriazine derivative, is one of the most widely used second-generation antiepileptic agents used for both partial and generalised seizures. In pregnant women, LTG is reported to produce side effects such as rash, mania, memory and cognitive problems, mood changes, runny nose, cough, nausea, indigestion, abdominal pain, weight loss, vaginitis, and leukopenia and retardation in development of fetus [8-10]. Recently, exposure to LTG during pregnancy was shown to impart adverse outcome within different developmental domains [11]. On the other hand its use in pregnancy is associated with risk of seizure deterioration, because its clearance is accelerated in pregnancy [12]. This reveals the need of additional information on the therapeutic window of LTG or its possible toxic effects in pregnancy.

Prenatal exposure to LTG (5-20 mg/kg/day) alone and in combination with MX-801, phenobarbital, or phenytoin resulted in cell death in the neonatal rat brain [13]. But, Katz et al. [14] showed no such effects in the same corresponding dose. Thus the effects of LTG during pregnancy, offspring 
development, and behaviour are still needed to be studied. The present study was undertaken to investigate the effects of prenatal exposure of LTG on postnatal development and its impact on offspring behaviour in rats.

\section{Materials and Methods}

2.1. Chemicals and Reagents. LTG was a kind gift from $\mathrm{M} / \mathrm{s}$ Sun Pharmaceutical Pvt. Ltd., Mumbai, India. Carboxymethyl cellulose (CMC) was procured from Himedia, Mumbai. PCR master cycler gradient was purchased from Genet Bio, Korea. TRIzol Reagent was purchased from Sigma Aldrich, USA. Unless mentioned, all other chemicals and reagents were of analytical grade.

2.2. Animal Husbandry and Ethics Approval. 20 male and 36 female Sprague Dawley rats were used for the study. Animals were housed in polypropylene cages in a wellventilated room (air cycles: $12-15$ air changes $/ \mathrm{min}$, recycle ratio: 70:30) under an ambient temperature of $23 \pm 2{ }^{\circ} \mathrm{C}$ and $40-65 \%$ relative humidity, with an artificial photoperiod of $12 \mathrm{~h}$ light/dark cycle. They were provided with rodent feed (Provimi Animal Nutrition India Pvt. Ltd.) and purified water ad libitum. Animals were acclimatized for a period of 7 days to the laboratory conditions prior to initiation of the experiment. Guidelines of "Guide for the Care and Use of Laboratory Animals" (Institute of Laboratory Animal Resources, National Academic Press 1996, NIH publication number 85-23, revised 1996) were strictly followed throughout the study. Institutional Animal Ethics Committee (IAEC), Sri Ramachandra University, Chennai, India, approved the study protocol (IAEC/XIV/SRU/99/2008).

2.3. Groups and Treatment. Following acclimatization, female rats were introduced with proven breeder during night time (18:00) and were isolated during day hours (09:00) in the ratio of $3: 1$, respectively, for copulation. Vaginal smear test was performed for each female rat every $24 \mathrm{~h}$ and the pregnancy were confirmed by the existence of diestrum stage for three consecutive days. The day of confirmation of pregnancy was designated as GND 3 and the pregnant rat was isolated into a separate cage. The dose of LTG was arrived from the maximum recommended human dose $(500 \mathrm{mg})$ in the management of epilepsy (http://www.drugs.com/).

Pregnant rats were randomized into 4 groups (6 in each) based on stratified body weight. Group-I received vehicle (0.5\% CMC; $10 \mathrm{~mL} / \mathrm{kg}$, p.o.); Group-II, III, and IV received LTG at $11.5,23$, and $46 \mathrm{mg} / \mathrm{kg}$, p.o., respectively (three-dose regimen was selected to investigate the dose dependent response). Weekly body weight and cumulative feed and water consumption were measured in dam. LTG was administered from GND 3 and continued till 11 days following parturition. Pregnant rats were allowed to deliver and wean their pups until PND 21. Male and female offspring were separated from dam on day 22 following delivery.

2.4. Physical Parameters. Length of gestation in dam and viability index of the pups were recorded. Litter sizes in each group were restricted to eight pups (4/sex). Body weight gain was measured on postnatal days once in a week till the study termination. The day of occurrence for pinna detachment, incisor eruption, eye opening, vaginal opening, and testes descent was also recorded.

2.5. Behavioural Function in Male and Female Offspring. Offspring were allowed to mature for 90 days with free access to food and water ad libitum. On PND 91, the animals were subjected to behavioural assessment.

2.5.1. Anxiety-Elevated Plus Maze. Anxiety was assessed in offspring using elevated plus maze [15]. Elevated plus maze was constructed of black painted wood with two open arms $(50 \times 10 \times 1 \mathrm{~cm})$ and two closed arms $(50 \times 10 \times 40 \mathrm{~cm})$ extending from a common central platform $(10 \times 10 \mathrm{~cm})$ and elevated to $45 \mathrm{~cm}$ above floor level. Experiments were carried out in a sound-attenuated, temperature-controlled room and illuminated by two $60 \mathrm{w}$ fluorescent light. On PND 91, rats were individually placed in the center of the maze facing open arm. Number of entries into open and closed and time spent in open and closed arms were recorded for a period of $5 \mathrm{~min}$ [16-19].

2.5.2. Locomotor Function-Open Field Exploratory Test. Locomotor behavioural assessment in experimental groups was performed using open field exploratory test [20]. Open field apparatus consisted of a plywood floor $(96 \times 96 \mathrm{~cm})$ with high walls. The entire apparatus was painted black except for $6 \mathrm{~mm}$ thick white lines which divided the floor into 16 squares. On PND 91, each animal was placed at one corner of the apparatus and observed for next $5 \mathrm{~min}$. The number of squares crossed, immobility period (in seconds), and number of rearing and grooming were recorded.

2.5.3. Learning- and Memory-Radial Arm Maze. Radial arm maze was made of black painted wood finished with a polyurethane coating and consisted of 8 arms with a center platform of $60 \mathrm{~cm}$ in diameter. The arms were $80 \mathrm{~cm}$ long and $15 \mathrm{~cm}$ wide. A disposable plastic cup was placed at each end of the arm to reinforce the sweet smash and all arms were baited with either a food cup or an empty cup. The entire radial arm maze was elevated $50 \mathrm{~cm}$ off the floor. Visual cues were located throughout the room to provide spatial cues. The maze was wiped down with $70 \%$ alcohol between each trail. The offspring were food-restricted to $85-90 \%$ and maintained for the duration of training and testing period.

The maze task was divided into three phases: adaptation, training, and retention phases. In adaptation phase, the rats were allowed to explore the radial arm maze by $3 \times 5 \mathrm{~min}$ trials. During this period, all of the arms of the maze were baited with the food so that the rats received experience in gaining a feed reinforcement by completely traversing an arm and reaching into the food cup. Time taken by the animal to move from the starting point (lag period), number of working memory errors, and time taken by the animal to munch the food in all arms were recorded. During the training period, four alternative arms were baited with food. 
TABLE 1: Effect of LTG on dam body weight and feed and water consumption.

\begin{tabular}{|c|c|c|c|c|}
\hline \multirow{2}{*}{ Treatment } & \multirow{2}{*}{ Control } & \multicolumn{3}{|c|}{ LTG (mg/kg, p.o) } \\
\hline & & 11.5 & 23 & 46 \\
\hline \multicolumn{5}{|c|}{ Dam body weight (g) } \\
\hline GND 3 & $182.00 \pm 6.00$ & $165.00 \pm 1.20$ & $169.83 \pm 3.56$ & $183.69 \pm 7.82$ \\
\hline GND 7 & $187.00 \pm 8.50$ & $171.67 \pm 1.64$ & $168.83 \pm 4.12$ & $180.50 \pm 4.85$ \\
\hline GND 14 & $227.50 \pm 13.25$ & $188.33 \pm 5.60^{*}$ & $185.53 \pm 5.64^{*}$ & $178.33 \pm 9.91^{* *}$ \\
\hline GND 21 & $242.00 \pm 8.50$ & $201.66 \pm 12.88^{*}$ & $191.00 \pm 9.16^{* *}$ & - \\
\hline \multicolumn{5}{|c|}{ Cumulative feed consumption (g/dam) } \\
\hline GND 3-6 & $40.75 \pm 2.37$ & $37.00 \pm 3.14$ & $39.29 \pm 1.37$ & $41.37 \pm 3.48$ \\
\hline GND 7-14 & $86.00 \pm 1.14$ & $60.28 \pm 4.26^{* *}$ & $58.00 \pm 2.09^{* *}$ & $47.56 \pm 0.96^{* *}$ \\
\hline GND 15-21 & $88.50 \pm 1.86$ & $65.25 \pm 2.50^{* *}$ & $60.80 \pm 1.52^{* *}$ & - \\
\hline \multicolumn{5}{|c|}{ Cumulative water consumption $(\mathrm{mL})$} \\
\hline GND 3-6 & $43.39 \pm 0.31$ & $41.25 \pm 2.32$ & $39.77 \pm 0.29$ & $44.56 \pm 1.55$ \\
\hline GND 7-14 & $102.50 \pm 0.59$ & $96.54 \pm 1.56^{*}$ & $95.80 \pm 1.09^{* *}$ & $91.00 \pm 1.33^{* *}$ \\
\hline GND 15-21 & $95.50 \pm 1.08$ & $86.59 \pm 2.03^{* *}$ & $85.80 \pm 1.17^{* *}$ & - \\
\hline
\end{tabular}

$n=6$ /group. Values are expressed in mean \pm SEM. Significance with Tukey's test following one way ANOVA is indicated as ${ }^{*} P \leq 0.05$ and ${ }^{* *} P \leq 0.01$ versus control group; GND: gestational day; LTG: lamotrigine.

The same arms were remained baited for all training and retention trials. Three 5-minute trials were conducted to each animal for ten days. The trial was terminated when the rat has entered and eaten from all the four baited arms. At the end of the three trials of each day, the rat was returned to its home cage. In retention period, the animal was tested $24 \mathrm{~h}$ after the final training trials. They received three trials as described under the training procedure. Lag time, number of reference memory errors, working memory errors, correct entries, and total time taken by the animal to munch the food were recorded [21].

2.6. GABA-A and GABA-B mRNA Expression by Reverse Transcriptase-Polymerase Chain Reaction (RT-PCR). After behavioural assessment, all the experimental animals were euthanized using $\mathrm{CO}_{2}$ exposure and cortical brain structures were collected for GABA-A and GABA-B mRNA expression. Briefly, the total RNA was extracted using TRIzol Reagent (Sigma, USA). After homogenization, the tubes were incubated for $10 \mathrm{~min}$ and centrifuged at $1000 \mathrm{rpm}$ for $5 \mathrm{~min}$. $200 \mu \mathrm{L}$ of chloroform was added to the supernatant, allowed to incubate for $5 \mathrm{~min}$ at room temperature, and centrifuged at $12000 \mathrm{rcf}$ for $20 \mathrm{~min}$. Then $500 \mu \mathrm{L}$ of isopropyl alcohol was added to the supernatant to precipitate total RNA and centrifuged at $12000 \mathrm{rcf}$ for $15 \mathrm{~min}$ following the incubation period of $10 \mathrm{~min}$. Supernatant was decanted carefully and pellet was washed thrice with $75 \%$ alcohol and centrifuged at $12000 \mathrm{rcf}$ for $15 \mathrm{~min}$ and the pellet was dried. The pellet was resuspended in RNase-free water and stored in $-80^{\circ} \mathrm{C}$ until use. The isolated RNA was allowed to undergo reverse transcription and polymerization reaction to get cDNA using RT-PCR master cycler gradient (Genet Bio, Korea). The gene expression was analyzed using the bands formed in agarose gel electrophoresis, captured using Gel documentation unit (Vilber Laumar, Germany) and quantified by Bio ID software.

Primers sequence used were as follows: GABA-A: sense, 5'-AAG GAC CCA TGA CAG TGC TC-3'; antisense, $5^{\prime}$-GGC TCC CTT GTC CAC TCA TA- ${ }^{\prime}$. GABA-B: sense,
$5^{\prime}$-GCT GGA TGG TTA CCA CAT AG-3 ${ }^{\prime}$; antisense $5^{\prime}$-GGT CAC AGG AGC AGT GAT G- $3^{\prime}$ and $\beta$-actin: sense, $5^{\prime}$-TGC TGT CCC TGT ATG CCT CT- $3^{\prime}$; antisense, $5^{\prime}$-AGG TCT TTA CGG ATG TCA ACG-3' [22].

2.7. Statistical Analysis. Data were expressed as mean \pm standard error of mean (SEM). Mean differences between the groups were analysed by one way ANOVA followed by Tukey's multiple comparison as post-hoc test. $P$ value $\leq 0.05$ was considered to be significant. Statistical analysis was performed using GraphPad prism 4.0 (San Diego, USA).

\section{Results}

3.1. Effect of LTG on Dam Body Weight and Feed and Water Consumption. Administration of LTG, at $46 \mathrm{mg} / \mathrm{kg}$, p.o, produced severe signs of toxicity such as hyperesthesia, vocalisation, recumbency, vaginal bleeding, nasal discharge, and finally death of the dam between GND 15 and 17. A significant decrease in body weight, food, and water consumption was observed in LTG (11.5 and $23 \mathrm{mg} / \mathrm{kg}$, p.o) administered dam on GND $14(F(3,20)=5.81, P<0.01$; $F(3,20)=43.11, P<0.01$ and $F(3,20)=15.51, P<0.01$, resp. $)$ and $21(F(2,15)=6.74, P<0.01 ; F(2,15)=55.23, P<0.01$ and $F(2,15)=13.08, P<0.01$, resp.) when compared to control (Table 1).

3.2. Effect of LTG on Physical Parameters in Offspring. LTG (11.5 and $23 \mathrm{mg} / \mathrm{kg}$, p.o) significantly increased $(F(2,15)$ $=23.83, P<0.01)$ the gestational period when compared to control rats. No significant difference in litter size between the groups was observed. However, a nonsignificant decrease in pups viability index was observed in LTG administered group when compared to control group (Table 2). LTG (11.5 and $23 \mathrm{mg} / \mathrm{kg}$, p.o) produced a significant delay in incisor eruption in both male $(F(2,15)=6.96, P<$ $0.05)$ and female $(F(2,9)=6.36, P<0.05)$ offspring when 
TABLE 2: Effect of LTG on length of gestation, total number of litters, and its viability index.

\begin{tabular}{lcccc}
\hline Group & Treatment & Gestational length (Days) & Total number of litters & Viability index (\%) \\
\hline I & Control & $19.33 \pm 0.33$ & $10.00 \pm 0.58$ & $100.00 \pm 0.00$ \\
II & LTG $(11.5 \mathrm{mg} / \mathrm{kg}$, p.o $)$ & $22.25 \pm 0.47^{* *}$ & $6.75 \pm 1.49$ & $80.20 \pm 15.90$ \\
III & LTG $(23 \mathrm{mg} / \mathrm{kg}$, p.o $)$ & $22.00 \pm 0.00^{* *}$ & $8.33 \pm 0.67$ & $81.48 \pm 13.36$ \\
IV & LTG $(46 \mathrm{mg} / \mathrm{kg}$, p.o $)$ & - & - & - \\
\hline
\end{tabular}

$n=6$ /group. Values are expressed in mean \pm SEM. Significance with Tukey's test following one way ANOVA is indicated as ${ }^{*} P \leq 0.05$ and ${ }^{* *} P \leq 0.01$ versus control group.

TABLE 3: Effect of LTG on physical growth of male and female offspring.

\begin{tabular}{|c|c|c|c|c|c|c|c|c|c|}
\hline \multirow{2}{*}{ Group } & \multirow{2}{*}{ Treatment } & \multicolumn{2}{|c|}{ Day of pinna detachment } & \multicolumn{2}{|c|}{ Day of incisor eruption } & \multicolumn{2}{|c|}{ Day of eye opening } & \multirow{2}{*}{$\begin{array}{c}\text { Day of testes } \\
\text { descent }\end{array}$} & \multirow{2}{*}{$\begin{array}{c}\text { Day of vaginal } \\
\text { opening }\end{array}$} \\
\hline & & Male & Female & Male & Female & Male & Female & & \\
\hline I & Control & $4.33 \pm 0.33$ & $4.67 \pm 0.33$ & $7.33 \pm 0.33$ & $7.33 \pm 0.33$ & $16.67 \pm 0.33$ & $16.00 \pm 0.33$ & $6.33 \pm 0.33$ & $32.67 \pm 0.33$ \\
\hline II & $\begin{array}{c}\text { LTG } \\
(11.5 \mathrm{mg} / \mathrm{kg}, \mathrm{p} . \mathrm{o})\end{array}$ & $5.33 \pm 0.33$ & $5.33 \pm 0.33$ & $9.33 \pm 0.33^{*}$ & $9.00 \pm 0.57$ & $15.66 \pm 0.66$ & $15.00 \pm 0.67$ & $6.23 \pm 1.67$ & $40.57 \pm 0.33^{* *}$ \\
\hline III & $\begin{array}{c}\text { LTG } \\
(23 \mathrm{mg} / \mathrm{kg}, \text { p.o })\end{array}$ & $5.30 \pm 0.29$ & $5.66 \pm 0.33$ & $9.50 \pm 0.64^{*}$ & $9.33 \pm 0.33^{*}$ & $15.33 \pm 0.33$ & $14.33 \pm 0.33$ & $9.00 \pm 0.00$ & $54.00 \pm 1.00^{* *}$ \\
\hline
\end{tabular}

$n=8$ /group (4/sex). Values are expressed in mean \pm SEM. Significance with Tukey's test following one way ANOVA is indicated as ${ }^{*} P \leq 0.05$ and ${ }^{* *} P \leq 0.01$ versus control group.

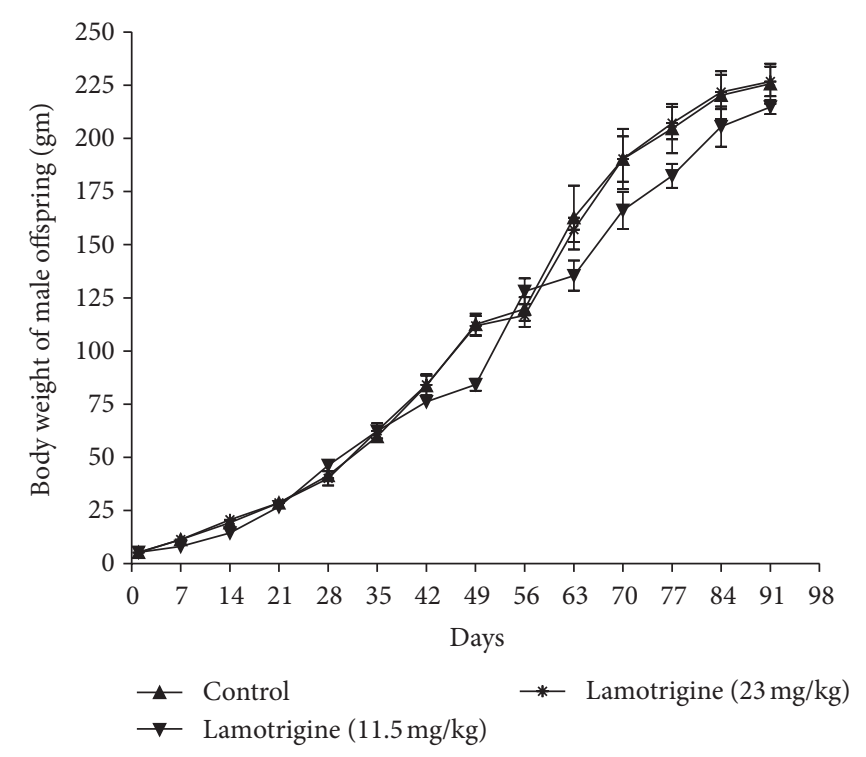

FIGURE 1: Effect of LTG on body weight of male offspring.

compared to control offspring. A similar observation was recorded in vaginal opening in female offspring $(F(2,9)=$ $286.5, P<0.01)$. There were no significant differences in the day of pinna detachment, eye opening, and testes decent observed between LTG and control offspring (Table 3). No significant difference in body weight was observed in both male and female offspring in comparison to control offspring throughout the study (Figures 1 and 2).

\subsection{Effect of LTG on Offspring Behaviour}

3.3.1. Anxiety-Elevated Plus Maze. LTG male showed no significant difference in number of entries and time spent

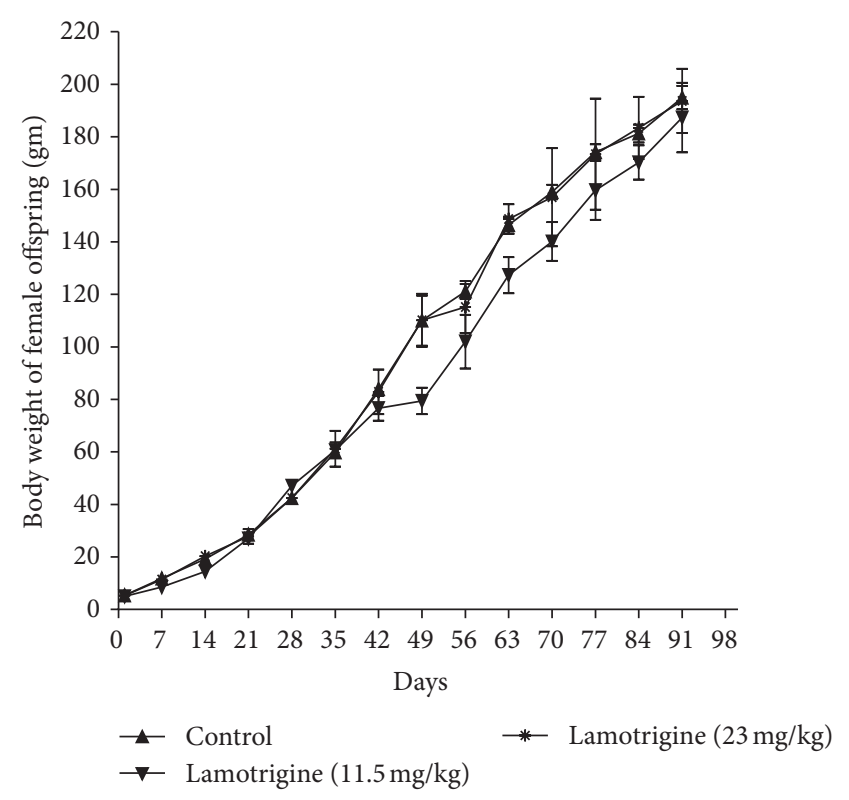

FIGURE 2: Effect of LTG on body weight of female offspring.

between open and closed arms. However, LTG female (23 mg/kg, p.o) showed a significant increase in number of entries $(F(2,9)=5.14, P<0.05)$ and time spent $(F(2,9)=$ 58.57, $P<0.01)$ in open arms and decreased number of entries $(F(2,9)=20.88, P<0.01)$ and time spent $(F(2,9)=$ 58.57, $P<0.01)$ in closed arms when compared to control rats (Table 4$)$.

3.3.2. Locomotor Function-Open Field Exploratory Test. No significant difference was observed in number of squares crossed, immobility period, and between the experimental groups. However, a significant $(F(2,9)=4.07$, 
TABLE 4: Effect of LTG on anxiety behaviour in male and female offspring.

\begin{tabular}{|c|c|c|c|c|c|c|}
\hline \multirow{2}{*}{ Group } & \multirow{2}{*}{ Treatment } & \multirow{2}{*}{ Sex } & \multicolumn{2}{|c|}{ Number of entries } & \multicolumn{2}{|c|}{ Time spent (sec) } \\
\hline & & & Open & Closed & Open & Closed \\
\hline \multirow{2}{*}{ I } & \multirow{2}{*}{ Control } & Male & $2.50 \pm 0.50$ & $10.25 \pm 1.18$ & $112.25 \pm 12.94$ & $187.75 \pm 12.94$ \\
\hline & & Female & $3.00 \pm 1.34$ & $11.00 \pm 0.84$ & $122.40 \pm 9.92$ & $177.60 \pm 9.92$ \\
\hline \multirow{2}{*}{ II } & \multirow{2}{*}{ LTG (11.5 mg/kg, p.o) } & Male & $2.08 \pm 0.94$ & $7.03 \pm 1.25$ & $133.72 \pm 28.13$ & $166.28 \pm 28.13$ \\
\hline & & Female & $4.67 \pm 1.00$ & $9.67 \pm 1.16$ & $97.92 \pm 16.89$ & $202.08 \pm 16.89$ \\
\hline \multirow{2}{*}{ III } & \multirow{2}{*}{ LTG (23 mg/kg, p.o) } & Male & $2.83 \pm 0.48$ & $8.00 \pm 0.73$ & $147.33 \pm 10.97$ & $152.67 \pm 10.97$ \\
\hline & & Female & $10.50 \pm 2.50^{*}$ & $3.50 \pm 0.50^{* *}$ & $262.50 \pm 4.50^{* *}$ & $37.50 \pm 4.50^{* *}$ \\
\hline
\end{tabular}

$n=8$ /group (4/sex). Values are expressed in mean \pm SEM. Significance with Tukey's test following one way ANOVA is indicated as ${ }^{*} P \leq 0.05$ and ${ }^{* *} P \leq 0.01$ versus control group.

TABLE 5: Effect of LTG on locomotor function in male and female offspring.

\begin{tabular}{lcccccc}
\hline Group & Treatment & Sex & Number of squares crossed & Immobility period (sec) & Number of rearing & Number of grooming \\
\hline \multirow{2}{*}{ I } & \multirow{2}{*}{ Control } & Male & $89.25 \pm 7.45$ & $100.25 \pm 21.99$ & $27.25 \pm 2.43$ & $8.25 \pm 1.25$ \\
& & Female & $109.60 \pm 7.78$ & $72.00 \pm 11.36$ & $37.60 \pm 4.95$ & $7.40 \pm 0.75$ \\
\multirow{4}{*}{ II } & LTG $(11.5 \mathrm{mg} / \mathrm{kg}$, p.o) & Male & $87.00 \pm 5.29$ & $74.50 \pm 13.82$ & $28.00 \pm 1.34$ & $6.17 \pm 0.54$ \\
& & Female & $109.50 \pm 6.50$ & $81.42 \pm 17.32$ & $46.50 \pm 3.50$ & $9.00 \pm 3.00$ \\
& & Male & $77.52 \pm 16.40$ & $112.03 \pm 41.11$ & $20.85 \pm 3.85$ & $6.12 \pm 1.07$ \\
III & \multirow{2}{*}{ LTG $(23 \mathrm{mg} / \mathrm{kg}$, p.o) } & Female & $113.58 \pm 4.64$ & $50.00 \pm 10.00$ & $51.75 \pm 1.00^{*}$ & $5.08 \pm 0.63$ \\
\hline
\end{tabular}

$n=8$ /group (4/sex). Values are expressed in mean \pm SEM. Significance with Tukey's test following one way ANOVA is indicated as ${ }^{*} P \leq 0.05$ versus control group.

$P<0.05)$ increase in rearing behaviour was observed in LTG (23 mg/kg, p.o) female offspring when compared to control group (Table 5 ).

3.3.3. Learning- and Memory-Radial Arm Maze. There was no significant difference in lag period, number of reference and working memory errors, number of correct entries, and total time taken by the animal to munch the food recorded between the experimental groups (Table 6).

3.4. Effect of LTG on GABA-A and GABA-B mRNA Expressions. mRNA expression of GABA-A was found to be significantly $(F(2,3)=17.07, P<0.05)$ downregulated in LTG female offspring (23 mg/kg, p.o) when compared to counterparts; whereas, no difference in GABA-B expression was observed between the experimental groups (Figure 3).

\section{Discussion}

Use of antiepileptic drugs, such as LTG and levetiracetam, during pregnancy has become challenging these days as they have toxic effects on the developing embryo. Several investigations in rat and rabbit models revealed that LTG crosses the placenta [23]. Administration of LTG in the form of single therapy or polytherapy is at a high risk of developing signs and symptoms of fetal toxicity [24]. The present study demonstrates that prenatal exposure of LTG in rats produced severe signs of toxicity in dam and gender differential behavior in female offspring.

Various studies demonstrated that prenatal LTG exposure at a dose of $250 \mathrm{mg} / \mathrm{kg}$ (half the human equivalent dose) increases stillbirth and postnatal death of the offspring $[25,26]$. In the current study, gestational LTG exposure at $46 \mathrm{mg} / \mathrm{kg}$ produced severe toxic signs such as hyperesthesia, vocalisation, recumbency, vaginal bleeding, nasal discharge, and finally death of the dam. Sidhu et al. [27] showed that lamotrigine administration increases the follicle stimulating hormone $(\mathrm{FSH})$ and luteinizing hormone ( $\mathrm{LH})$ which in turn stimulated estrogen secretion. Increased estrogen secretion results in maturation of graaffian follicle followed by ovulation which leads to embryo detachment. This may be the possible reason for the observed fetal death in the present study.

In addition, LTG induces the secretion of parathyroid hormone $(\mathrm{PTH})$ thereby modulating calcium homeostasis leading to osteoporosis [28]. It is well known that osteogenesis of the embryo occurs at GND 15-21 in rats. Increased serum calcium levels by PTH reduce the fetal osteogenesis during embryonic development which could also be one of the possible factors for observed fetal death at GND 15-17 in LTG high dose administered rats. Decreased body weight gain and feed and water intake of dam treated with LTG revealing its possible maternal toxic effects. Further, delayed vaginal opening and incisors eruption in offspring of LTG lower doses group might also be due to its maternal toxic effects [13].

On the other hand, it is reported that LTG administration along with the estrogen-containing hormonal contraceptives reduces the serum LTG concentration. Hence the dose has to be increased when administered with such contraceptives to maintain its level of action [29]. In addition, increased estrogen level induces FSH and secretes progesterone and testosterone by feedback control process thereby lengthening the 
TABLE 6: Effect of LTG on learning and memory function in male and female offspring.

\begin{tabular}{lccccccc}
\hline Group & Treatment & Sex & Lag period & $\begin{array}{c}\text { Number of reference } \\
\text { memory errors }\end{array}$ & $\begin{array}{c}\text { Number of } \\
\text { working } \\
\text { memory errors }\end{array}$ & $\begin{array}{c}\text { Number of } \\
\text { correct } \\
\text { entries }\end{array}$ & $\begin{array}{c}\text { Total time taken to } \\
\text { munch the food }\end{array}$ \\
\hline I & \multirow{2}{*}{ Control } & Male & $1.00 \pm 0.00$ & $1.83 \pm 0.80$ & $0.42 \pm 0.12$ & $2.25 \pm 0.74$ & $82.50 \pm 19.04$ \\
& & Female & $0.33 \pm 0.20$ & $0.73 \pm 0.37$ & $0.20 \pm 0.13$ & $2.53 \pm 0.51$ & $88.93 \pm 15.81$ \\
II & LTG (11.5 mg/kg, p.o) & Male & $1.06 \pm 0.41$ & $2.67 \pm 0.44$ & $0.28 \pm 0.08$ & $1.22 \pm 0.43$ & $137.11 \pm 15.54$ \\
& & Female & $0.32 \pm 0.10$ & $2.67 \pm 0.74$ & $1.17 \pm 0.50$ & $1.33 \pm 0.89$ & $113.17 \pm 15.00$ \\
& & Male & $1.25 \pm 0.33$ & $3.00 \pm 0.74$ & $0.71 \pm 0.17$ & $1.25 \pm 0.53$ & $141.25 \pm 15.68$ \\
III & LTG (23 mg/kg, p.o) & Female & $0.47 \pm 0.22$ & $1.46 \pm 0.72$ & $0.75 \pm 0.43$ & $2.33 \pm 0.69$ & $63.71 \pm 19.24$ \\
\hline
\end{tabular}

$n=8 /$ group $(4 /$ sex $)$. Values are expressed in mean \pm SEM.
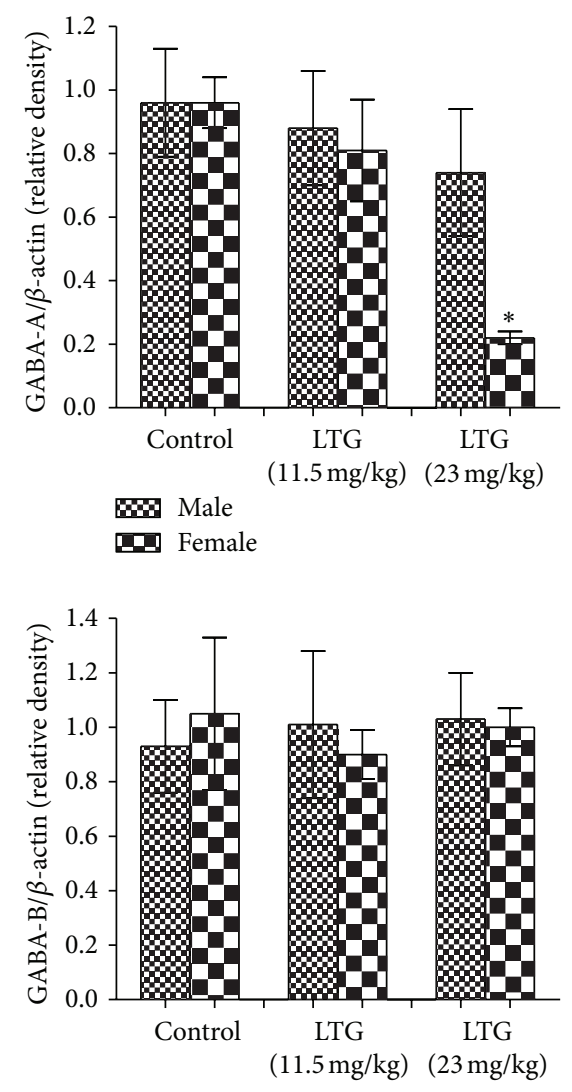

WOA Male

므 Female

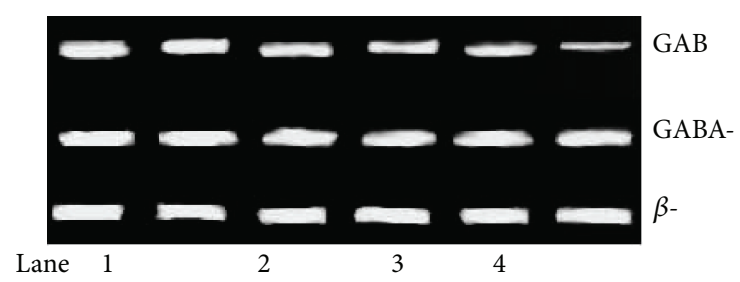

FIgURE 3: Effect of LTG on GABA receptors in male and female offspring. Lane 1- and 2-Control (male and female offspring, resp.); Lane 3- and 4-LTG: $11.5 \mathrm{mg} / \mathrm{kg}$, p.o (male and female offspring, resp.); Lane 5- and 6-LTG: $23 \mathrm{mg} / \mathrm{kg}$, p.o (male and female offspring, resp.). ${ }^{*}$ denotes $P \leq 0.05$ versus control group. gestational period. Although progesterone and testosterone levels were not measured in dams, the increased gestational length observed in our study of rats who administered LTG at lower doses may be due to impaired luteolysis and increased progesterone levels.

Earlier studies have shown that blockade of GABAA receptor in rat brain induces hyperexcitability/anxiolytic behaviour in elevated plus maze $[30,31]$. Progesterone and estrogen induce anxiolytic behaviour in C57BL/ 6 female mice [32]. Further, estrogen suppresses GABA-A receptor expression thereby slowing down the GABA mediated inhibition [33]. In the present study, the observed decrease in GABA-A expression and hyperactivation behaviour (as evidenced by the elevated plus maze and open field test) may be due to the overactivation of G-protein-coupled receptor 30 (GPR30) by estrogen hormone in the female offspring. Put together, the observations showed that the anxiolytic behaviour in female offspring but not the male may be due to the defect in GABAA expression and also the alterations in GPR30 mediated estrogen secretion.

\section{Conclusion}

In conclusion, the present study demonstrates the potential untoward effects of LTG in pregnant rats and also its influence on the postnatal development and gender based differential behavioural effects in offspring. Our lab is further investigating the mechanisms and reason for these untoward effects so as to utilise the therapeutic benefits of LTG in a safer way.

\section{Abbreviations \\ AED: Antiepileptic drug \\ LTG: Lamotrigine \\ GND: Gestational day \\ PND: Postnatal day}

GABA-A: $\gamma$-Aminobutyric acid A receptor

GABA-B: $\gamma$-Aminobutyric acid B receptor

CMC: Carboxymethyl cellulose

IAEC: Institutional Animal Ethics Committee

RT-PCR: Reverse transcriptase polymerase chain reaction

SEM: $\quad$ Standard error of mean

PTH: Parathyroid hormone 
FSH: Follicle stimulating hormone

LH: $\quad$ Luteinizing hormone

GPR30: G-protein-coupled receptor 30 .

\section{Conflict of Interests}

The authors declare no conflict of interests.

\section{Acknowledgments}

The authors thank Drugs and Pharmaceutical Division, Department of Science and Technology, Govt. of India, for funding the project. Authors express their sincere thanks to Prof. Dr. S. Thanikachalam, Director, Centre for Toxicology and Developmental Research (CEFT), Sri Ramachandra University (SRU), for providing the facilities to carry out the project. Authors extend their thanks to all technical and nontechnical staff of CEFT-SRU, Chennai, for their help during conduct of the experiment.

\section{References}

[1] L. B. Holmes, "The teratogenicity of anticonvulsant drugs: a progress report," Journal of Medical Genetics, vol. 39, no. 4, pp. 245-247, 2002.

[2] K. J. Meador, G. A. Baker, R. H. Finnell et al., "In utero antiepileptic drug exposure: fetal death and malformations," Neurology, vol. 67, no. 3, pp. 407-412, 2006.

[3] E. Perucca, "Birth defects after prenatal exposure to antiepileptic drugs," The Lancet Neurology, vol. 4, no. 11, pp. 781-786, 2005.

[4] N. Adab, A. Jacoby, D. Smith, and D. Chadwick, "Additional educational needs in children born to mothers with epilepsy," Journal of Neurology Neurosurgery and Psychiatry, vol. 70, no. 1, pp. 15-21, 2001.

[5] J. Vinten, N. Adab, U. Kini, J. Gorry, J. Gregg, and G. A. Baker, "Neuropsychological effects of exposure to anticonvulsant medication in utero," Neurology, vol. 64, no. 6, pp. 949-954, 2005.

[6] J.-B. Manent, I. Jorquera, I. Mazzucchelli et al., "Fetal exposure to GABA-acting antiepileptic drugs generates hippocampal and cortical dysplasias," Epilepsia, vol. 48, no. 4, pp. 684-693, 2007.

[7] D. Janz and U. Fuchs, "Are anti epileptic drugs harmful during pregnancy?” Deutsche Medizinische Wochenschrift, vol. 89, pp. 241-248, 1964.

[8] B. R. Danielsson, K. Lansdell, L. Patmore, and T. Tomson, "Effects of the antiepileptic drugs lamotrigine, topiramate and gabapentin on hERG potassium currents," Epilepsy Research, vol. 63, no. 1, pp. 17-25, 2005.

[9] V. Des Portes, I. Souville, F. Francis et al., "So-called "cryptogenic" partial seizures resulting from a subtle cortical dysgenesis due to a doublecortin gene mutation," Seizure, vol. 11, no. 4, pp. 273-277, 2002.

[10] K. J. Meador, G. A. Baker, N. Browning et al., "Cognitive function at 3 years of age after fetal exposure to antiepileptic drugs," The New England Journal of Medicine, vol. 360, no. 16, pp. 1597-1605, 2009.

[11] G. Veiby, A. K. Daltveit, S. Schjolberg et al., "Exposure to antiepileptic drugs in utero and child development: a prospective population-based study," Epilepsia, vol. 54, no. 8, pp. 14621472, 2013.
[12] C. T. Clark, A. M. Klein, J. M. Perel et al., "Lamotrigine dosing for pregnant patients with bipolar disorder," The American Journal of Psychiatry, vol. 170, no. 11, pp. 240-247, 2013.

[13] J.-B. Manent, I. Jorquera, V. Franco, Y. Ben-Ari, E. Perucca, and A. Represa, "Antiepileptic drugs and brain maturation: fetal exposure to lamotrigine generates cortical malformations in rats," Epilepsy Research, vol. 78, no. 2-3, pp. 131-139, 2008.

[14] I. Katz, J. Kim, K. Gale, and A. Kondratyev, "Effects of lamotrigine alone and in combination with MK-801, phenobarbital, or phenytoin on cell death in the neonatal rat brain," Journal of Pharmacology and Experimental Therapeutics, vol. 322, no. 2, pp. 494-500, 2007.

[15] S. Pellow and S. E. File, "Anxiolytic and anxiogenic drug effects on exploratory activity in an elevated plus-maze: a novel test of anxiety in the rat," Pharmacology Biochemistry and Behavior, vol. 24, no. 3, pp. 525-529, 1986.

[16] E. Appenrodt, R. Schnabel, and H. Schwarzberg, "Vasopressin administration modulates anxiety-related behavior in rats," Physiology and Behavior, vol. 64, no. 4, pp. 543-547, 1998.

[17] S. E. Bowen, J. L. Wiley, and R. L. Balster, "The effects of abused inhalants on mouse behavior in an elevated plus-maze," European Journal of Pharmacology, vol. 312, no. 2, pp. 131-136, 1996.

[18] E. F. Espejo, "Effects of weekly or daily exposure to the elevated plus-maze in male mice," Behavioural Brain Research, vol. 87, no. 2, pp. 233-238, 1997.

[19] T. Skutella, J. C. Probst, U. Renner, F. Holsboer, and C. Behl, "Corticotropin-releasing hormone receptor (type I) antisense targeting reduces anxiety," Neuroscience, vol. 85, no. 3, pp. 795805, 1998.

[20] R. G. Lister, "Ethologically-based animal models of anxiety disorders," Pharmacology and Therapeutics, vol. 46, no. 3, pp. 321-340, 1990.

[21] D. S. Olton, C. Collison, and M. A. Werz, "Spatial memory and radial arm maze performance of rats," Learning and Motivation, vol. 8, no. 3, pp. 289-314, 1977.

[22] T. Tamayama, K. Maemura, K. Kanbara et al., "Expression of GABAA and GABAB receptors in rat growth plate chondrocytes: activation of the GABA receptors promotes proliferation of mouse chondrogenic ATDC5 cells," Molecular and Cellular Biochemistry, vol. 273, no. 1-2, pp. 117-126, 2005.

[23] P. Prakash, L. V. Prabhu, M. A. Nasar, R. Rai, S. Madhyastha, and G. Singh, "Lamotrigine in pregnancy: safety profile and the risk of malformations," Singapore Medical Journal, vol. 48, no. 10, pp. 880-883, 2007.

[24] P. A. Forcelli, M. J. Janssen, L. A. Stamps, C. Sweeney, S. Vicini, and K. Gale, "Therapeutic strategies to avoid long-term adverse outcomes of neonatal antiepileptic drug exposure," Epilepsia, vol. 51, no. 3, pp. 18-23, 2010.

[25] GenRx, The Complete Reference for Generic and Brand Drugs, Mosby, St Louis, Mo, USA, 9th edition, 1999.

[26] T. Tomson, E. Perucca, and D. Battino, "Navigating toward fetal and maternal health: the challenge of treating epilepsy in pregnancy," Epilepsia, vol. 45, no. 10, pp. 1171-1175, 2004.

[27] J. Sidhu, S. Job, S. Singh, and R. Philipson, "The pharmacokinetic and pharmacodynamic consequences of the coadministration of lamotrigine and a combined oral contraceptive in healthy female subjects," British Journal of Clinical Pharmacology, vol. 61, no. 2, pp. 191-199, 2006.

[28] H. A. Valsamis, S. K. Arora, B. Labban, and S. I. McFarlane, "Antiepileptic drugs and bone metabolism," Nutrition and Metabolism, vol. 3, article 36, 2006. 
[29] A. Reimers, G. Helde, and E. Brodtkorb, "Ethinyl estradiol, not progestogens, reduces lamotrigine serum concentrations," Epilepsia, vol. 46, no. 9, pp. 1414-1417, 2005.

[30] C. H. Bueno, H. Zangrossi Jr., and M. B. Viana, "The inactivation of the basolateral nucleus of the rat amygdala has an anxiolytic effect in the elevated T-maze and light/dark transition tests," Brazilian Journal of Medical and Biological Research, vol. 38, no. 11, pp. 1697-1701, 2005.

[31] S. K. Sanders, S. L. Morzorati, and A. Shekhar, "Priming of experimental anxiety by repeated subthreshold GABA blockade in the rat amygdala," Brain Research, vol. 699, no. 2, pp. 250-259, 1995.

[32] K. M. Olesen, N. Ismail, E. D. Merchasin, and J. D. Blaustein, "Long-term alteration of anxiolytic effects of ovarian hormones in female mice by a peripubertal immune challenge," Hormones and Behavior, vol. 60, no. 4, pp. 318-326, 2011.

[33] M. J. Morrell, “Epilepsy in women," American Family Physician, vol. 66, no. 8, pp. 1489-1494, 2002. 

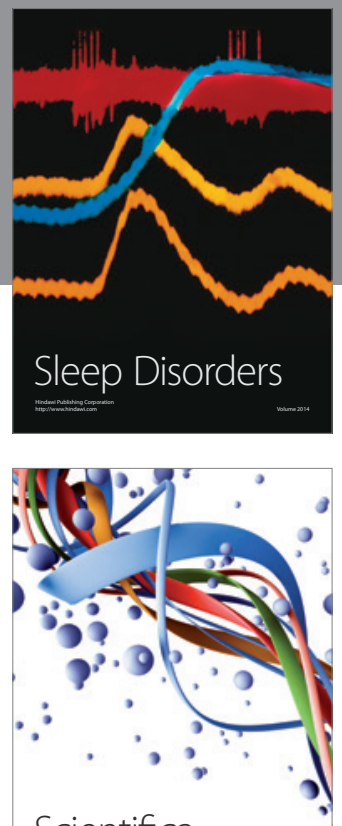

Scientifica
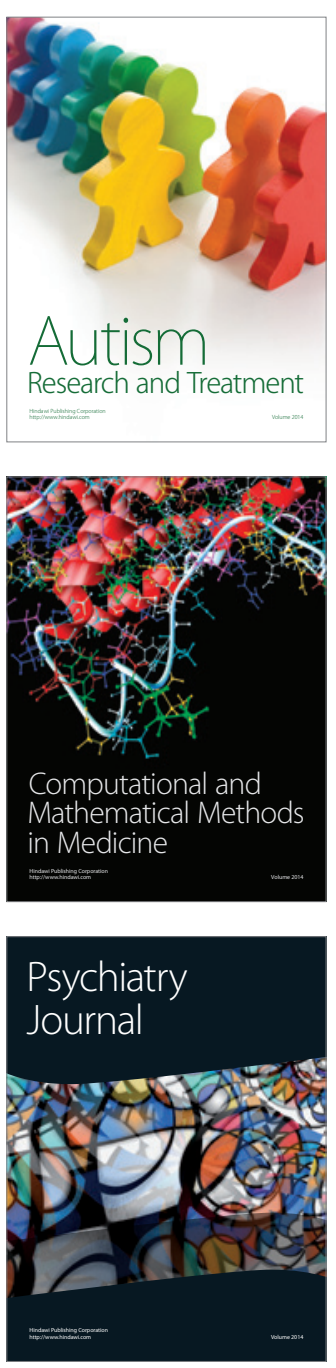
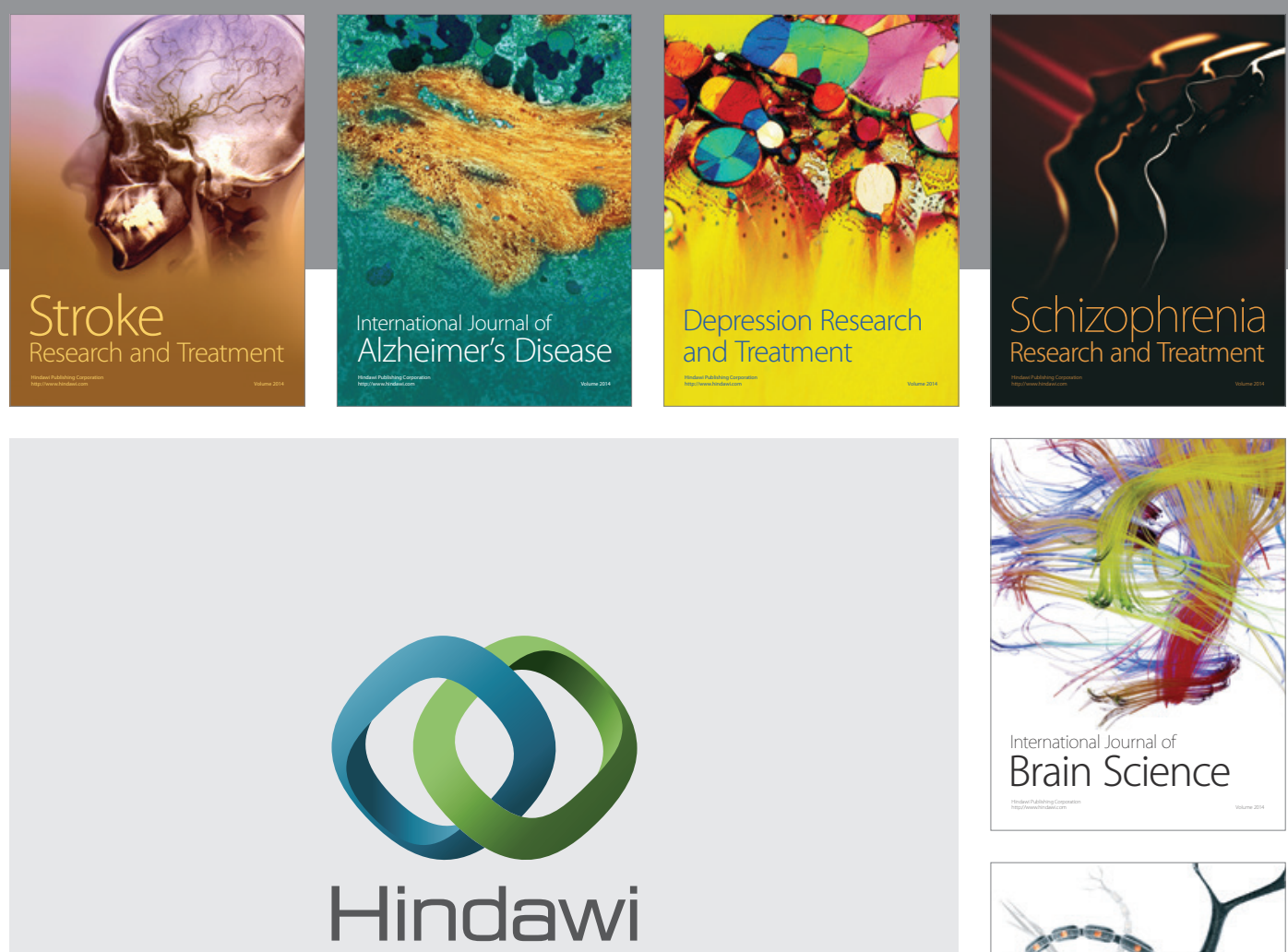

Submit your manuscripts at

http://www.hindawi.com
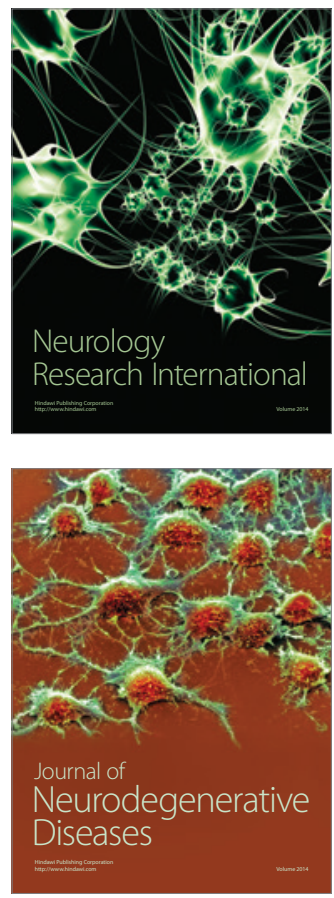

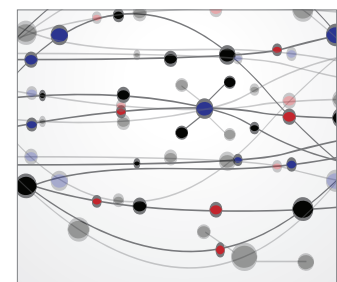

The Scientific World Journal
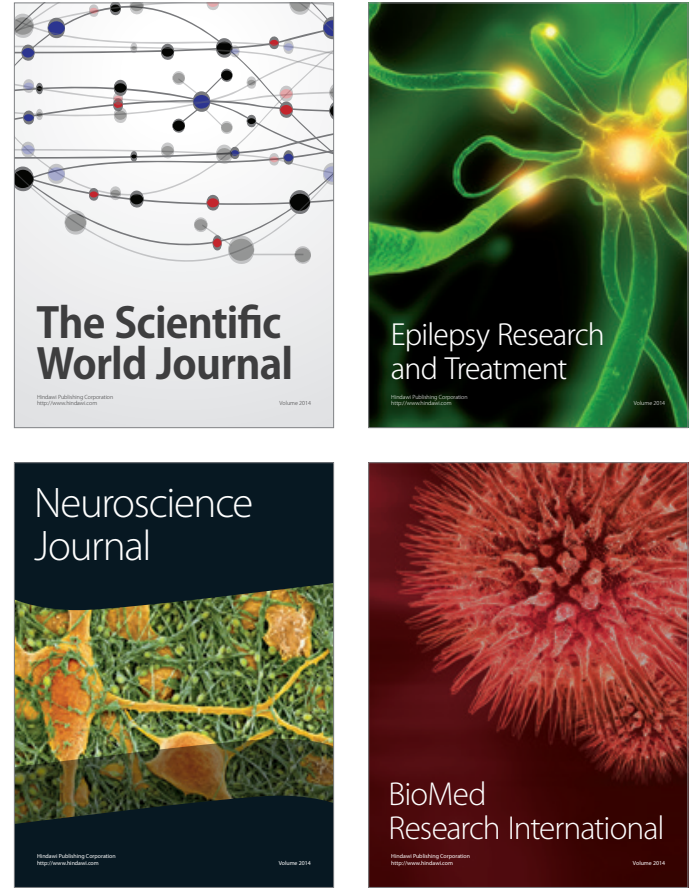

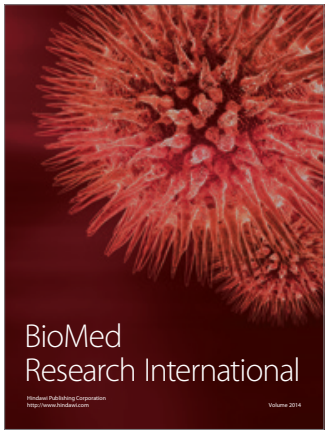

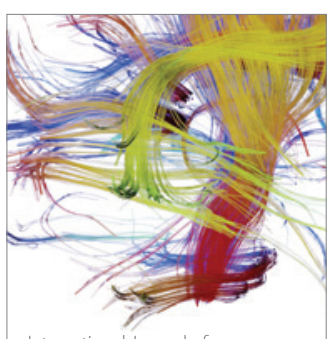

Brain Science

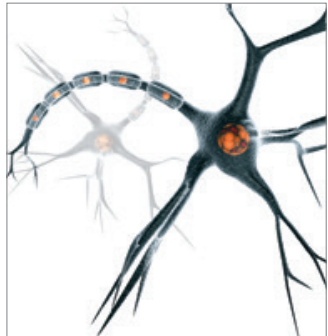

Neural Plasticity
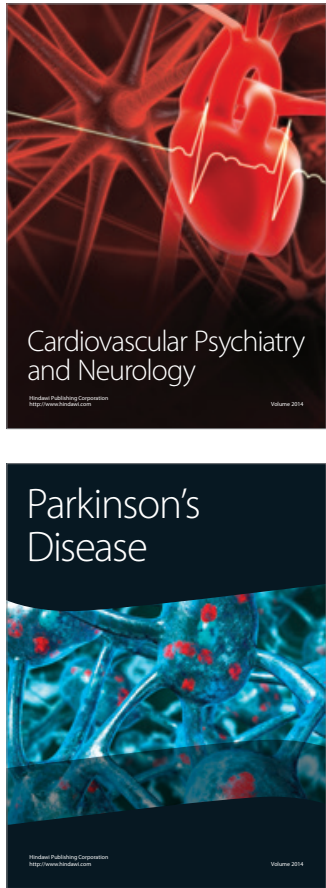